\title{
Making your point: principles of visual design for computer aided slide and poster production
}

\author{
Rob Forsyth, Alan Waller
}

This is the second article in a series on medical education.

Visual 'literacy' is an important skill for medical professionals to acquire if they are to communicate effectively with audiences of ever increasing visual sophistication. Full mastery of the techniques involved requires a long apprenticeship in graphic and print design, yet today's desktop publishing and presentation software put great design power into inexperienced hands. In a limited space, we hope to convey some of the principles of good visual design relevant to the use of such software in the production of slides and poster sets, while avoiding the specifics of any one software package or hardware platform.

Good visual design aims to ease communication: it should unobtrusively facilitate an enjoyable flow of information from presenter to viewer, leaving the latter free to concentrate on the content of the presentation. The flexibility of modern graphics software allows the use of previously unavailable techniques that can enhance legibility and accessibility; inappropriately used, however, they will irritate and distract from the content of the presentation.

An essential ingredient for successful communication lies in knowing your audience. You will have already considered listeners' knowledge of your field when deciding verbal aspects of your presentation such as the choice of vocabulary: it should also be keyed to visual aspects. Anyone who is in any way unfamiliar with your subject matter (usually most of your audience) will need signposts into and through the material in clear visual language, or they may abandon the attempt to keep up. A concern to provide a clear visual structure to your material must underlie the design process. Every design choice of your presentation - each

\section{Serif}

Times Roman

Palatino

Garamond

\section{$\Sigma \psi \mu \beta 0 \lambda$ (Symbol)}

Figure 1 Examples of popular serif and sans serif fonts suitable for use in slide and poster sets, together with Symbol for mathematical characters. See text for suggested applications. font choice, each graphic element - should be making an identifiable contribution to the clarity of the message; if it is not, it should go.

The main visual elements of any presentation will be text, illustrations, and graphs. We consider each in general terms before considering issues specific to slide or poster production.

\section{Text}

Text will be a central part of any slide or poster set. Fonts have individual personalities (fig 1) and some are more appropriate to formal presentations than others. Many presentation packages supply the user with predefined templates that make sensible typographical choices about font and size. If, however, you are using a general purpose word processing or graphics package to produce material, the choice is left to you.

Confusion surrounds usage of the word 'font'. In this paper we use it in the (technically inaccurate) sense in which it is often used in graphics program menus to mean a family of typefaces (for example, 'Times') of several styles (for example Times Roman, Times Bold and Times Italic), each in many sizes. The temptation to select widely from the font smorgasbord offered by your system should be resisted: it will lead to distraction. It should not be necessary to use more than two fonts - one serif and one sans serif - in a slide or poster set, plus Symbol or a similar font for special characters (fig 1).

Most software packages measure type size and leading (the spacing between lines) in the traditional printer's unit of 'points', defining a point as 1/72 inch. The small tails or 'serifs' on the ends of letters of serif fonts (fig 2) are intended to aid reading by visually linking adjacent letters. This makes them especially suitable for use in longer blocks of body text. Sans serif fonts such as Helvetica have a clarity and emphasis about them that makes them especially useful for titles and side headings, and perhaps the short points of a well designed slide. Long pieces of text set in a sans serif font tend to be harder to read.

Smooth reading requires that the eye assimilates whole words at a time, recognising them by their overall shapes. Considerable typographical experience and design evolution 


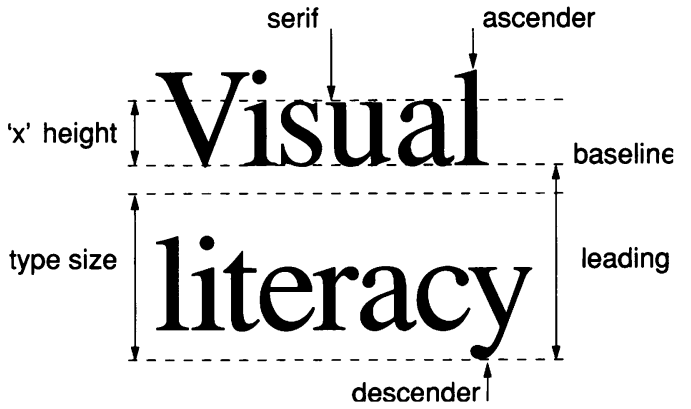

Figure 2 The anatomy of type. Default settings for most packages give a leading of $120 \%$ of the type size which is suitable for many purposes, though consider setting large point sizes (such as text in poster title banners) 'solid' that is, with a leading of $100 \%$.

A nlane nertlina to lattar fromon anhonnas tho

Figure 3 We do not assimilate the whole of every character when reading fluently. The upper half of characters and the shapes formed by the ascenders above the $x$ height line convey much of the needed information. The corresponding bottom half of this line is much less informative. This efficiency is lost in ALL CAPITALS setting, requiring more detailed inspection and loss of reading fluency.

has gone into maximising font legibility to facilitate the rapid scanning of whole lines of text at a time (fig 3). Font designers do, however, make certain assumptions about the usage of their creations. For example, capital ('upper case') letters are designed, as their name implies, to stand alone at the head of a sentence and not to be used for the setting of whole words or sentences: their design results in such text losing legibility. In the days of the typewriter, capitalisation and underlining were the only ways text could be emphasised, but neither technique meets the typographical criterion of enhanced legibility. Text should instead be emphasised using letter weight (for example, bold face) or colour. The legibility of italic text varies between fonts, but tends to be lower: it should be used sparingly, for single words and Latin names. Two other typographical habits can also betray the typewriter trained. Capitals are designed for use with a single rather than a double space after a full stop. Similarly, typists were obliged to use inch symbols (") for reported speech rather than the distinct opening and closing typographer's quotation marks (" ") that are available in most computer fonts.

Smooth scanning movements of the eye along successive lines of text is aided by keeping to a line length ('measure') of 50-70 characters (including spaces and punctuation) per line. Shorter lines interrupt scanning and the eye can have difficulty locating the start of the next line if measures are longer. Similarly, the varying starting position of lines of centred text disrupt scanning; centred text is suitable for titles, but should be avoided for body text. Fully justified text (flush on both left and right margins) can be a mixed blessing. Well executed, it conveys an overall visual impression of order to a poster, but poorly justified text is considerably worse than left justified ('ragged right') text. Some packages can only justify lines by adjusting word or letter spacing, which can be visually distracting if done clumsily (fig 4). Hyphenation can improve spacing, but some automatic hyphenation algorithms result in inappropriate word breaks. If you want to fully justify text, be prepared to invest time in manually adjusting hyphenation, letter spacing ('kerning'), and line breaks to optimise readability.
Font choice has a marked impact on the overall "personality" of a piece. Type size and leading also affect the colour of the page: the light or heavy feel conveyed by the total amount of

\begin{tabular}{l}
\hline FONT CHOICE HAS A MARKED \\
IMPACT ON THE OVERALL \\
“PERSONALITY" OF A PIECE. \\
TYPE SIZE AND LEADING ALSO \\
AFFECT THE COLOUR OF THE \\
\hline
\end{tabular}

Font choice has a marked impact on the overall "personality" of a piece. Type size and leading also affect the colour of the page: the light or heavy feel conveyed by the total

Font choice has a marked impact on
the overall "personality" of a piece.
Type size and leading also affect the
colour of the page: the light or heavy
feel conveyed by the total amount of

Font choice has a marked impact on the
overall "personality" of a piece. Type size
and leading also affect the colour of the
page: the light or heavy feel conveyed by the
total amount of ink on the page.

Figure 4 Unintentional loss of legibility in body text caused by inexperienced use of typographical techniques. Compare (in rows, starting from top left) normal left justified (ragged right) text with: all capitals; centred text; poorly executed full justification; use of font with inappropriate 'personality' (Zapf Chancery); and italic setting. 

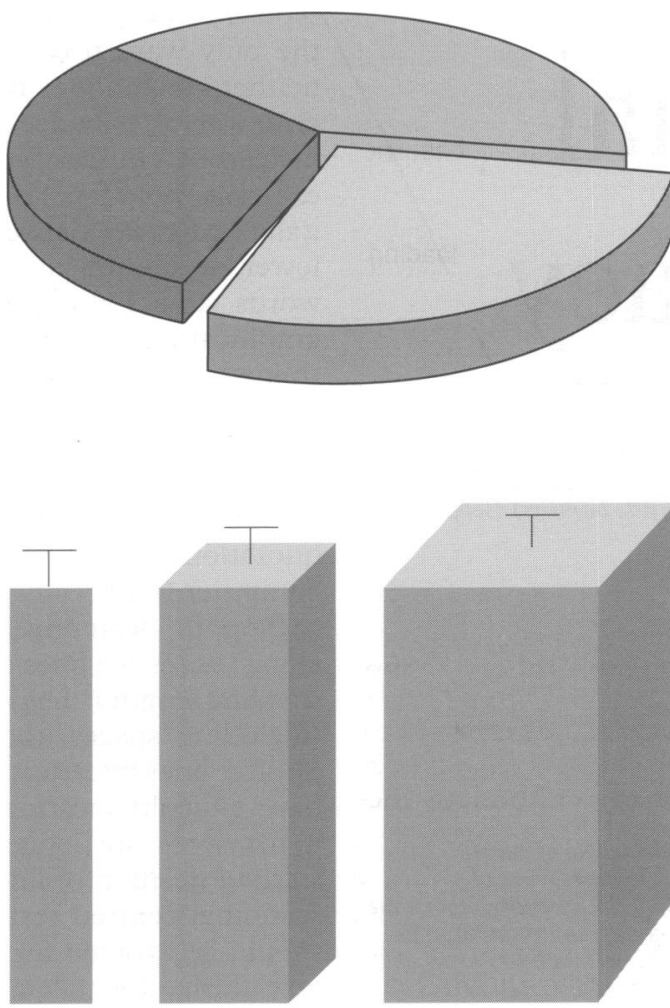

Figure 5 The evils of presentation package 'pseudo-3D' graph designs. The highlighted wedge of the pie chart is actually only $25 \%$ of the total. And how to make error bars appear smaller by adding 'depth' to a conventional two dimensional histogram column (left) so that the top of the error bar appears 'below' the rear edge of the column (centre and right).

\section{Graphs}

'Business presentation' packages are intended for salesmen trying to impress rather than inform. In particular, the use of 'pseudo-3D' graph designs (that is, two dimensional histogram or pie charts with added 'depth') can be downright misleading, and has little place in scientific presentations (fig 5).

Elements on a single graph that require distinction, such as histogram bars or pie
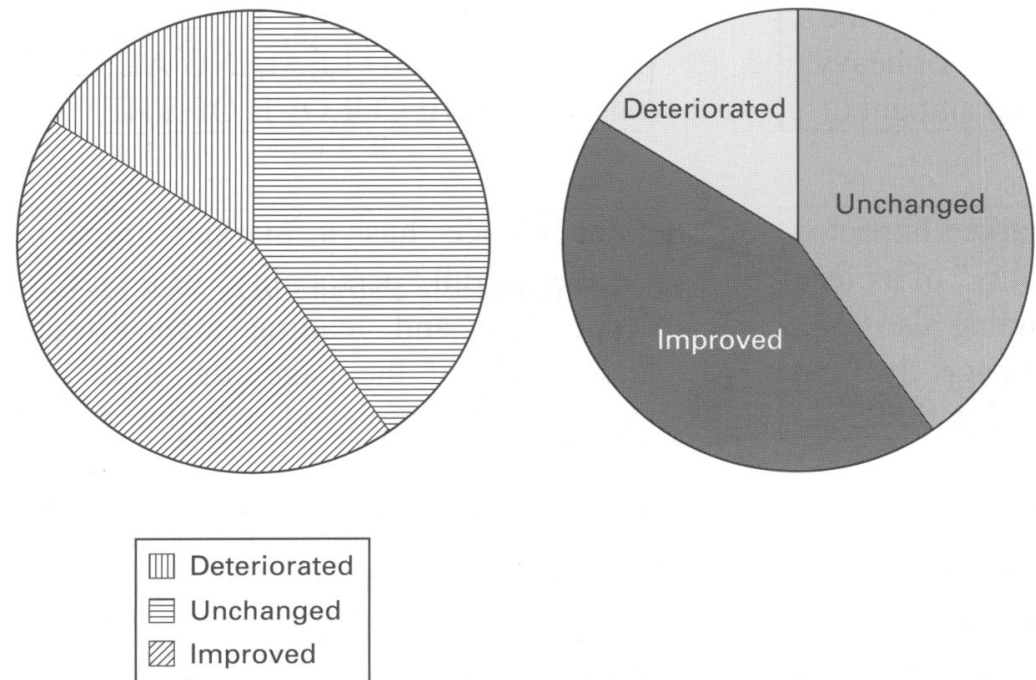

Figure 6 A less extreme case of bad graph design typical of the output of many graphing packages. The hatching patterns are not readily distinguishable, and the separate legend requires the viewer to look back and forth. In simple graphs, direct labelling of the wedges (right) enables a now redundant legend to be dispensed with. Tonal values are more immediately distinguished than hatching patterns of similar 'colour'. chart wedges, are usually more effectively differentiated using colour or greyscale values rather than hatching patterns (fig 6). If making full colour slides, however, bear in mind that it's actually hard to select more than five or six colours that are readily mutually distinguishable under projection conditions.

A common fault in the creation of slides is the incorporation of graphs that initially appeared in journals. Leaving copyright issues to one side, the design choices made by the journal's printers when preparing a graph for print will be inappropriate for use in projected images which must be designed for viewing at a much greater distance. Axis labels and numbers, and the size of the data point plot symbols will all be too small. Redraw graphs increasing the size of these elements relative to the axes themselves. Similarly, direct photocopying of tables from journals usually results in illegible slides.

\section{Illustrations}

Although we all know how many words a picture is worth, illustrations (as distinct from graphs) remain under used and undervalued in presentations, though they can add considerably to an audience's understanding. A flow chart can replace a verbose description of a clinical trial protocol; a schematic diagram can convey theoretical concepts. Good illustrations depend ultimately on an excellent knowledge of the subject at hand, and also ideally on some experience of which elements cause confusion when explaining it to others. Be willing to sacrifice minor details to enable a simplified, schematic treatment. Experiment on colleagues with back of envelope sketches to see which approach works; be creative and try to think of novel approaches to your topic.

Illustrations are probably best tackled in dedicated drawing packages and then pasted into a presentation package, whose own drawing tools are often fairly rudimentary. Certainly, if you anticipate producing flow diagrams, it is well worth learning to use a simple drawing package rather than trying to produce them on a word processor, where attempts to produce aligned blocks of text using tab characters rapidly degenerate into uneditable confusion. The more advanced illustration packages have steep learning curves, but their flexibility (such the ability to convey the impression of three dimensional structure using perspective, blends, and shading) can be useful. Bear in mind the comments made above in the context of graphs, that slides require text annotations in diagrams to be large relative to the illustration.

\section{Slide presentations}

Whereas the poster presentation has to grab the attention of a passer by, slide presentations are in general given to a captive audience. They can, however, still fall asleep! Remember that each graphic element on each slide must have earned its place because it enhances the communication of the presentation's message: 


\section{The bulleted slide}

- This line 36pt text on A4 page

- Needs a full minute to be taken in

- Points in note form

- One line per point

- Maximum seven lines per slide

- Check legibility at six times longest dimension

Figure 7 The basic bulleted slide. The original was produced on an $A 4$ paper suitable for conventional photography. The heading is 50 point Helvetica Bold, and the body text 36 point Times Roman with bold and italic emphases. Italics can be highly effective if used sparingly; however, the italic forms of some sans serif faces (such as Helvetica) are not so distinctive, and thus not as effective, as the Times Italic, shown here.

resist the temptation to use your presentation package's 'template' feature to add an element (such as your institution's logo) to every slide.

The two basic methods for slide production are direct output of a computer generated image to film in a film recorder, or traditional photography on a copying stand of laser printed paper output. If printing to paper, remember that the aspect (height to width) ratio of a $35 \mathrm{~mm}$ transparency and a piece of A4 paper are similar, so spread out and use all the available space. Unless your material demands a 'portrait' (tall) orientation, produce your slides in a 'landscape' (wide) format: you are less likely to be caught out by the edge of the image falling off the screen or being invisible to sections of the audience in a badly designed lecture theatre.

A related decision is whether the slide set will be monochrome (white lines on a black background) or full colour. Monochrome slides are produced from A4 printed output by conventional photography using negative line film; directly computer driven film recorders can use full colour film. Diazo films producing white on a coloured (typically blue) background are becoming less common. Although full colour slides are becoming increasingly widespread, don't dismiss monochrome as passé before considering its advantages. It's less affected by poor blackout in the lecture hall and it's often cheaper. The turnaround time may also be shorter, making correction of the spelling mistake you notice the day before the presentation feasible: check local details.

Unless your data would be greatly clarified by the ability to use full colour, seriously consider sticking to monochrome. The

\section{Where can I find what it's about?}

Where do I find the take-home message?

Where do I begin reading?

How far through am I?

Where's the summary?

Figure 8 Questions your design should enable a reader to answer about your poster. decision to use colour greatly increases the scope for design disasters and on-screen appearances differ from those of a projected slide in a darkened theatre in ways that can surprise the novice. Again, most templates provided by presentation packages make sensible choices. Use quiet dark blues and greens for the background, and white, light blue, and especially yellow for foreground text. A common mistake is the use of red to try to highlight important items. It typically appears surprisingly muted in projected slides. Highlight in white or yellow instead. Avoid the 'Las Vegas' look of large areas of pinks, mauves, or oranges which will distract from the content of the slide - and usually induce nausea - and bear in mind that nearly $10 \%$ of a male audience will be red-green colour blind! Develop a design that would still work if viewed in black and white.

If printing to a piece of A4 paper, use 24 point or larger type. You will find this significantly limits the amount of text you can fit on, and this is a vital discipline. Less, as ever, is more. If you can't fit it on one slide, split it between two, or better still, be more succinct. Don't plan to show more than one slide per minute of the presentation (fig 7). Use brief phrases on the slide and keep the script of your presentation in your hand, not on the screen.

\section{Posters}

Conference organisers are increasingly turning to the poster session as a means of accommodating ever greater numbers of papers and so poster preparation is an increasingly important skill. Posters have the advantage of being highly flexible, suitable for the display of text, photographs, tables, graphs, and select bibliographies. Normally conference organisers make few stipulations about form other than to specify an overall maximum size, leaving considerable scope for visual creativity.

A sadly common sight is a poster comprising many monochrome laser printed A4 sheets each on their own piece of coloured card. This results in an often confusing impression of separate islands of information, each apparently demanding equal attention. The situation is often aggravated by a failure to align these islands. The eye is very aware of edges, and the haphazard arrangement of the borders repeatedly distracts gaze from the text. Worse still, the text is often only legible from a few feet away.

The primary aims of the design must be to attract attention among a sea of posters competing for viewers' time, and then to enable the viewer to decide rapidly whether the poster is relevant or interesting. Visual structure must be provided for the viewer who is not going to study the poster in detail to pick out the key components (fig 8). These are in order of importance: the title, the aims and conclusion, then method, results, and discussion.

The use of the IMRAD (introduction, method, results, and discussion) structure used in papers may occasionally be specified by 


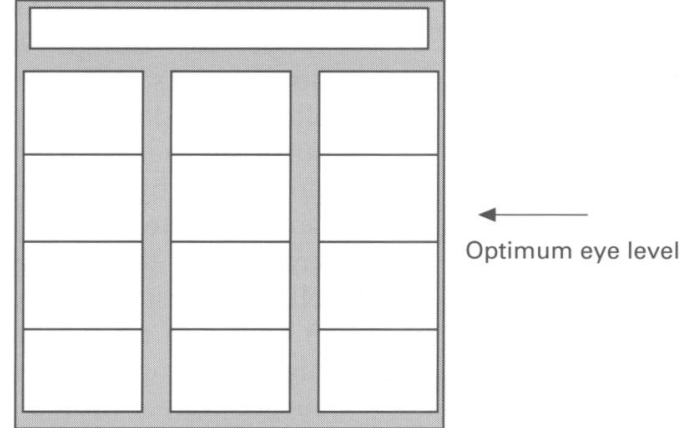

Figure 9 Poster layout using A3 spreads. This scale diagram shows how $12 \mathrm{A3}$ spreads (each $42 \mathrm{~cm} \times 29.7 \mathrm{~cm}$ ) fit onto a $150 \mathrm{~cm} \times 150 \mathrm{~cm}$ square poster area, with room for a $140 \mathrm{~cm} \times 15 \mathrm{~cm}$ title banner. This approach will need to be adapted to the poster area provided by the conference organiser. Note also the suggested ideal mounting height relative to the viewer's eye level.

conference organisers, and in any case has the advantage of familiarity so that viewers can find their way around the poster. Alternatively, you may want to use a more informal sequence of sections with more informative section titles. Keep titles succinct, in the active rather than the passive voice, and use them to convey conclusions as well as aims. Replace 'Pharmacological mediators of carcinogenesis in a rodent model' with ' $\mathrm{X}$ causes cancer in rats'.

Although many illustration packages will allow the design and layout of a 'page' of typical poster dimensions on screen, few generally available printers can handle paper larger than A3 size. There are two solutions. The theoretically more versatile, but more complex, alternative is to design the poster as a whole at full size on screen and then use the 'tiling' facility of many software packages to send slightly overlapping A3 sized portions of the whole image to the printer. These then have to be trimmed and assembled by hand onto a full sized supporting board. In practice, however, joins are difficult to hide, especially if running through blocks of text, and, in any case, the board will need to be cut up again into transportable sections. The alternative, which we prefer, is to design separate A3 spreads, perhaps using a desktop publishing package. With no elements crossing the boundaries of each A3 spread, this inevitably restricts possible design solutions. The greatly simplified printing saves time and headaches, however. A3 sheets are about the largest size conveniently carried in a briefcase, and a typical conference poster space allocation will accommodate 12 A3 spreads. Arranged in three columns of four, a natural reading order down each column in

Typographical recommendations for a poster presentation

\begin{tabular}{lllll}
\hline Section & $\begin{array}{l}\text { Reading distance } \\
\text { (feet) }\end{array}$ & $\begin{array}{l}\text { Type size } \\
\text { (points) }\end{array}$ & Font style & Weight \\
\hline Title & $15-20$ & $72-84$ & Sans serif or serif & Bold \\
Author and affiliations & $15-20$ & $60-72$ & Serif & Bold \\
Section headings & $6-8$ & $36-48$ & Sans serif (contrasting colour?) & Bold \\
Body text & $3-4$ & 24 & Serif & Regular \\
Figure legends & $3-4$ & 24 & Sans serif or serif &
\end{tabular}

*Select a complementary pair of fonts, one serif, one sans serif, and use them throughout. turn from left to right immediately suggests itself to the viewer, and this can be utilised (fig 9). We use a film laminator (available in medical art departments or high street print shops) to encapsulate each A3 sheet in a protective clear plastic, resulting in a robust yet flexible sheet that can be mounted directly on the conference poster board without requiring an additional card support.

A3 colour laser printers provide an economical and convenient means of full colour production and are becoming increasingly available in large institutions. Alternatively, commercial bureaux can print files generated by many popular packages from disk. Although colour match between screen and printer may not be perfect, the use of colour in printed output in general holds fewer unpleasant surprises than in slide production and, used effectively, makes a major contribution to the appeal of the poster. Consider the use of a muted but distinctive background wash such as a beige or light blue-grey to identify and unify the components of your poster and distinguish it from others. A distinctive colour for subheadings can also be effective. If only A4 monochrome printers are available, consider designing your poster in A4 spreads and using a photocopier to enlarge each to A3 before adding colour manually with marker pens and the like.

The table gives some typographical recommendations suitable for the title, subtitles, and body text of a poster. At the very large point sizes used for the title, the rules used to control the automatic spacing (kerning) between letter pairs and the default leading between lines (fig 2) both tend to generate too much white space between letters and lines. The appearance is improved by tightening kerning (if you have the facility) and reducing leading to $100 \%$ or slightly less. Not withstanding our comments above, you may want to consider using 'tiling' to produce a title banner that spans the full width of the poster, unifying the other elements (fig 9). This banner can also be laminated and the result will be flexible enough to be rolled loosely for transport.

The text of the poster should be considered carefully, and rigorously edited. Posters cannot be read and reread at leisure like a paper. If there are technical details that are only going to be of interest to specialists, consider producing a handout for them and freeing up poster space. There is much to be said for including a little light relief in a poster: something related to the subject but undemanding, such as a colour photograph of a piece of equipment, or better still some human interest. Consider including fax or phone numbers on the poster, or even providing a small dispenser of business cards, to encourage colleagues to contact you.

As a final comment, may we encourage you to seek out local advice. The medical illustration department of your local hospital will enjoy meeting people who appreciate the importance of good design and will be happy to offer information on local facilities. We look forward to seeing an increasing standard of visual literacy in future slide and poster presentations! 